\title{
Interview avec un membre de l'ASP Esther Bulang
}

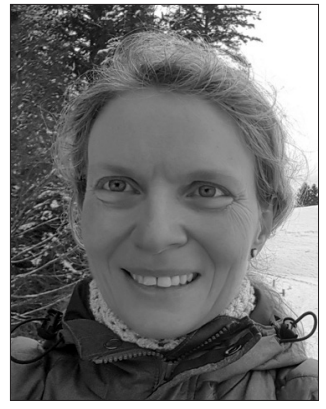

Esther Bulang
Quelles ont été vos motivations pour choisir le métier de psychothérapeute?

Je voulais placer lêtre humain dans sa globalité le travail sur et avec lêtre humain et une perspective holistique - au centre de mon activité professionnelle. Je voulais réellement avoir du temps pour les individus, savoir ce qui les intéresse, savoir comment ils pensent, ce qu'ils ressentent, ce qu'ils veulent. J'ai beaucoup réfléchi à ce qui est important pour moi dans la vie et à ce qui correspond le mieux à ma personnalité, à quel emploi et à la façon dont je souhaite le faire, à ce qui me rend heureuse et avec quoi je pourrais le mieux faire entendre ma voix. Pouvoir être psychothérapeute est pour moi vivre une philosophie de la vie.

Quel est votre contexte/parcours professionnel et quelle est sa signification pour votre activité professionnelle?

Directement après le baccalauréat, il était obligatoire dans l'ex-RDA de travailler, avant les années de médecine, comme auxiliaire de soins pendant un an dans un hôpital. S'en sont suivies mes années de médecine à Leipzig, elles se sont terminé la même année que la chute du mur en 1989. Aujourd'hui encore, le bouleversement lancé à l'époque à Leipzig me relie encore profondément à ce lieu. Le mémoire obligatoire pendant le cursus, pour mon cas un mémoire scientifique de type et de portée, n'a pas été reconnu comme thèse de doctorat pour des raisons politiques. Après mes études, devenue mère entre-temps de deux magnifiques enfants, j'ai obtenu une formation en cinq ans de médecin spécialiste en ophtalmologie et j’ai travaillé depuis lors comme tel dans le secteur clinique. En 2003, j'ai obtenu alors un doctorat dans la spécialité Neurologie/neuro-ophtalmologie à l'Université Technique de Dresde. Lobtention du doctorat a été ponctuée de voyages de conférence, également aux USA, une expérience extrêmement importante.

Lorsqu'en 2006, ma décision est tombée de devenir psychothérapeute à part entière, jétais arrivée au summum de ma réussite en tant que chef d'une petite clinique ophtalmologique et en tant qu'opératrice en microchirurgie intraoculaire. À ce moment, j'avais opéré des yeux presque quotidiennement pendant près de onze ans. Mon succès reposait, en plus de la réussite opératoire, principalement sur le fait que javais appris à intégrer dans l'activité structurée et rationnelle mon sentiment pour les gens que jopérais et pour toute mon équipe et que je moccupais des gens d'une manière très appliquée et individuelle. Quelque chose dont les personnes âgées en particulier qui ont besoin d'aide sont très reconnaissantes. La plus vieille patiente que j’ai opérée avait 97 ans. Mais j’ai aussi remarqué que les longues journées d'opérations avec une posture corporelle uniforme au microscope opératoire me réservaient de plus en plus de douleurs corporelles et jétais à la recherche d'une vision sur la façon dont je voulais aménager mes années professionnelles avec une bonne qualité de vie jusquau départ en retraite. Dans la mesure où ni le secteur stationnaire avec sa rentabilisation croissante ni le secteur ambulatoire avec une forte charge de travail et peu de temps pour les patients me proposaient une situation professionnelle satisfaisante, $j$ 'avais en quelque sorte atteint le point final de mon activité professionnelle. Jusqu'à présent, j'avais aidé les gens à un niveau physique à avoir une meilleure vue, il devait maintenant s'agir de rendre lâme des gens plus voyante. Pendant environ deux ans, en complément professionnel de mon activité, jai commencé ma formation à l'Institut de Dresde de Psychothérapie Psychodynamique avec une participation régulière aux séminaires du soir et le début d'une analyse didactique. Auparavant comme médecin en chef avant la prise de la direction générale de la clinique, j’ai obtenu de la direction de la clinique un mois de disponibilité non rémunérée et j'ai travaillé comme chirurgien en ophtalmologie et comme formatrice d'une nouvelle méthode opératoire dans une clinique ophtalmologique au Kenya, à l'extérieur de Nairobi, puis également dans un engagement de courte durée comme flying doctor dans un hôpital de brousse. Le respect de l'altérité et de l'acceptabilité de ne pas pouvoir sauver tout le monde, de respecter les limites de mes possibilités en ce qui concerne la vie et la santé des autres font entre autres partie des expériences les plus marquantes de ce travail. J'ai célébré le passage de ma vie d'ophtalmologue à celle de psychothérapeute par un voyage de quatre semaines, trek en haute montagne à pied depuis le Népal vers le Tibet, et au Tibet la circumambulation, la Kora, de la montagne sacrée Kailash et la visite de Lhassa. Une expérience profondément spirituelle. 
J'ai alors passé en Allemagne le deuxième diplôme de spécialiste en "Médecine psychosomatique et psychothérapie ». Car, pour moi il était évident dès le début du passage à l'activité psychothérapeutique que je voulais devenir psychothérapeute corporelle, l'absence de possibilités de formation en Allemagne m'a amené en Suisse. Mon ancien chef de clinique avait activement soutenu cette formation. En Suisse, j'ai terminé en sept ans, en complément professionnel à la formation psychanalytique de psychologie des profondeurs et psychodynamique en Allemagne, une formation très intense en psychothérapie corporelle à l'Institut de Biosynthèse à Heiden auprès de David et Silvia Boadella.

Ensuite, j’ai effectué pendant un an et demi une formation de thérapeute en tir à l'arc thérapeutique, qui comprend entre autres de réfléchir, de manipuler une arme et de discerner aussi d'une manière très consciente les énergies féminines et masculines en moi. Depuis 2014, je suis en formation et formation continue en thérapie des traumatismes avec la spécialisation en traumatisme et troubles dissociatifs auprès d'Ellert Nijenhuis. Une formation de superviseur était également incluse dans la biosynthèse et la thérapie des traumatismes selon Nijenhuis. J'ai le droit aussi d’enseigner les deux. J'ai réalisé un intérêt très personnel et un rêve présent depuis longtemps, en continuant à me former dans des formes thérapeutiques complémentaires et en me consacrant au thème de la spiritualité dans la vie et dans la psychothérapie. Depuis neuf ans, je suis une expérience de soi et une formation en chamanisme après de la chamane coréenne Hiah Park et auprès du chaman groenlandais Angaangaq Angakkorsuaq ainsi que depuis plus de 13 ans, une formation complète en bouddhisme tibétain et en techniques thérapeutiques tibétaines bouddhistes. J'ai également effectué une formation en massage énergétique Kum Nye et en psychologie bouddhiste, je suis enseignante de yoga tibétain de guérison Lu Jong et je termine cette année ma formation d'enseignante de méditation tibétaine bouddhiste auprès de Lharampa Tenzin Kalden.

En plus de l'analyse didactique, de la formation en psychothérapie corporelle et en thérapie des traumatismes, la formation chamanique est pour moi la découverte de soi la plus profonde. Également parce que j’appartiens à une petite minorité slave occidentale, les wendes-sorbes, qui vivent en Allemagne de l'Est dans le voisinage de la Pologne et de la Tchéquie. Cette quête d'identité comporte aussi la recherche de mes racines avant la christianisation et l'assimilation de la culture allemande.

Mon activité professionnelle sélève à seulement $50-70$ pour cent. Je renonce ainsi sciemment à des choses de la vie quotidienne que je pouvais avant moffrir financièrement, je vis plus modestement et d'une manière plus consciente. Je ne pourrais pas être heureuse si en plus d'une activité professionnelle à 100 pour cent, il ne me restait pas de temps pour l'engagement dans des domaines sociétaux, comme la protection du climat et l'environnement.

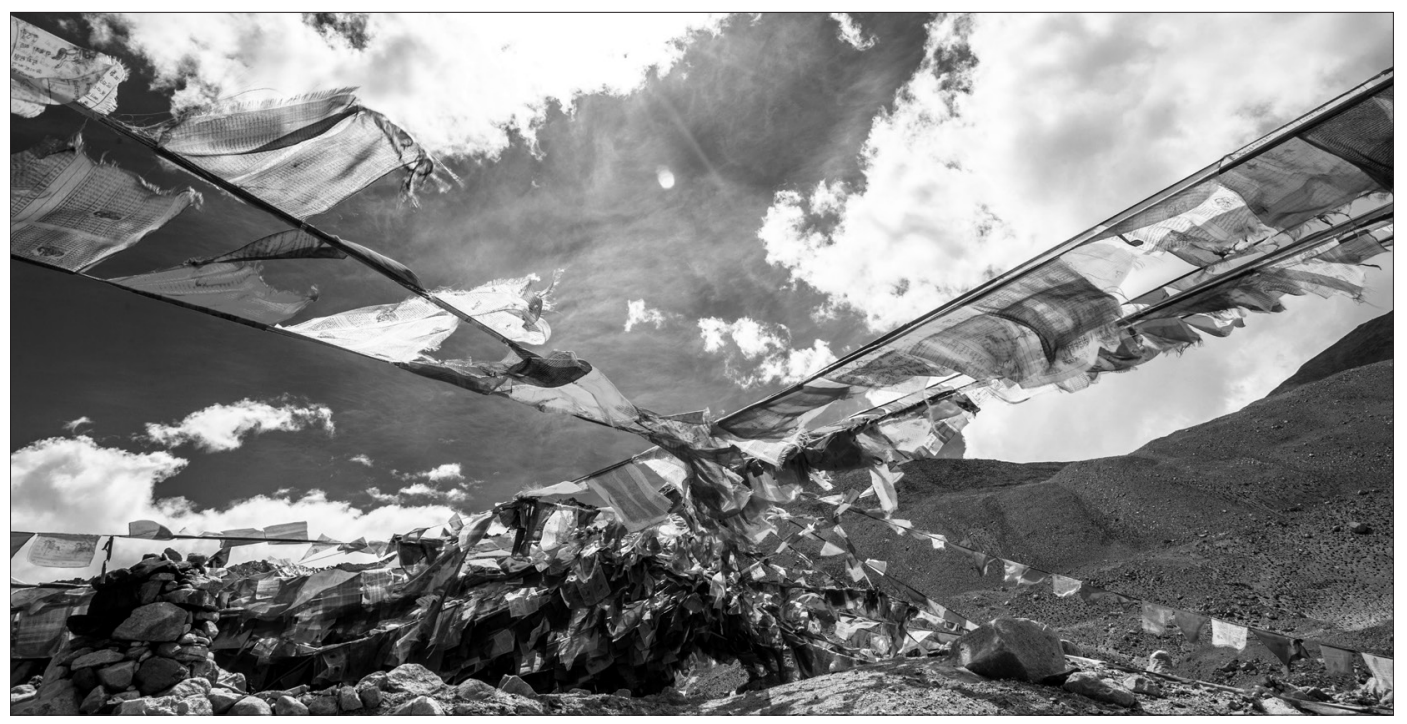


Travaillez-vous en tant que psychothérapeute indépendante dans un cabinet libre et/ou travaillez-vous encore en plus en tant que psychothérapeute déléguée?

En raison de mes formations et diplômes en Allemagne et en Suisse, je suis employée dans un cabinet médical, principalement en tant que psychothérapeute indépendante avec la reconnaissance de la SAPPM et je peux aussi, grâce à la reconnaissance de la FMPP (deux attestations d'exercer pour les médecins en Suisse), déléguer en psychothérapie. En outre, j'ai un propre cabinet dans lequel je travaille en tant que psychothérapeute indépendante.

Y a-t-il encore une autre profession, une autre occupation que vous exercez en plus de la psychothérapie?

Dans le cabinet médical, je travaille aussi dans une moindre mesure sur le plan de lassistanat médical dans la médecine complémentaire (médecine orthomoléculaire).

Dans mon propre cabinet, je souhaiterais bientôt proposer le yoga tibétain de guérison, Lu Jong, le massage tibétain Kum Nye, la méditation tibétaine bouddhiste et si possible, le tir à l'arc thérapeutique ainsi que le travail énergétique de guérison. Pour pouvoir le réaliser, il y a avant tout un aspect financier, donc cela progresse lentement et selon les possibilités existantes. Un site Internet est en cours de construction.

\section{Quelles sont ici les motivations?}

Pour ce qui est du yoga de guérison et de la méditation, je le fais, d'une part, pour moi, c'est un bel équilibre. Et en même temps, c'est l'expression de ma philosophie holistique pour lềtre humain et les patients. On ne peut pas tout intégrer dans une heure de psychothérapie et il existe tellement de belles choses qui peuvent nous aider mentalement et physiquement à rester ou à être en bonne santé et à pouvoir vivre en étant satisfait.

\section{Quelle est votre spécialisation?}

Mes spécialisations sont la thérapie des traumatismes, en particulier des traumas chroniques et les troubles dissociatifs (thérapie énactive des traumatismes selon Nijenhuis), la biosynthèse psychothérapie corporelle selon David et Silvia Boadella, ainsi que les états de conscience modifiés et la spiritualité. Également en association avec mon activité médicale de thérapie complémentaire, le traitement du burnout.

Vous sentez-vous satisfaite de votre situation professionnelle?

Globalement, je me sens très satisfaite de ma situation, car j’ai trouvé ce quoon appelle une «niche » pour mon activité de psychothérapeute dans laquelle j'ai le sentiment quon me traite équitablement dans une relation d'employée et que je peux exercer en complète indépendance mon activité de psychothérapeute dans le cadre de l'assurance obligatoire. Cela signifie que je peux en toute autonomie définir l'indication concernant la psychothérapie et donner aux gens qui en ont besoin en ce moment le soutien de psychothérapeute sans formalités complexes. Cela m’est possible grâce à ma double reconnaissance en tant que médecin et psychothérapeute. Je suis très reconnaissante que le système suisse le permette. En même temps, j'ai aussi conscience que cela est possible ainsi pour moi uniquement dans cette " niche ». En recherchant cette place, j'ai été beaucoup confrontée aux problèmes des psychothérapeutes psychologiques dans une relation de travail chez un ou une psychiatre. S'il existe certainement aussi de bonnes relations de travail entre les psychiatres et les psychothérapeutes psychologiques, l'impression perdure que les psychothérapeutes ne vont souvent pas bien dans cette relation de dépendance que je ne pourrais pas accepter dans la mesure entendue. Je ne sais pas si je pourrais gagner actuellement assez en tant que psychothérapeute libérale dans mon cabinet indépendant dans la métropole de Zurich pour subvenir à mes besoins. Et cela me met mal à l'aise que je ne puisse pas aider, avec ma qualification en tant que psychothérapeute libérale, les gens dans la même proportion, par exemple lorsque le complément des mutuelles est bientôt arrivé à expiration et qu'il y a des gens qui auraient besoin en urgence d'une psychothérapie, mais qu'ils ne peuvent pas continuer à la payer de leurs poches parce que lassurance obligatoire ne finance pas l'activité libérale des psychothérapeutes. Je suis ravie que le système suisse nait fondamentalement pas restreint, avec l'application de la loi sur les professions relevant du domaine de la psychothérapie, la diversité de la psychothérapie. Et je suis extrêmement reconnaissante et je remercie, en plus de l'OFS, l'association des psychothérapeutes l'ASP de sêtre mobilisée et d'avoir agi. 
Y a-t-il quelque chose que vous souhaiteriez être différent?

Je souhaite qu'après la création de mesures homogènes pour la formation des psychothérapeutes psychologiques avec la loi sur les professions relevant du domaine de la psychothérapie, ces derniers puissent travailler en libéral pour l'assurance obligatoire en tant que groupe professionnel indépendant. Si le système se fait confiance et fait confiance à son travail et que chaque école reconnue de psychothérapie est pleinement consciente de la responsabilité de sa formation et y fait face, cela devrait être absolument possible. Je souhaite aussi qu'il existe beaucoup plus d'acceptation des postes à temps partiel et ainsi créer beaucoup plus d’opportunités pour les gens qui souhaitent travailler ainsi.

Je souhaite par ailleurs qu'il y ait plus d'ouverture impartiale associée à de la curiosité, de l'acceptation et du respect par rapport aux méthodes complémentaires, ce qui signifierait aussi en particulier de reconnaitre la spiritualité comme un facteur actif d'efficience dans la psychothérapie. Je souhaite que le secteur de la psychothérapie, en tant que secteur indépendant, souvre davantage aux disciplines voisines et que des réseautages naissent, par ex. avec la philosophie, les sciences culturelles ou naturelles comme la physique quantique à partir desquelles la psychothérapie peut déduire des principes universels de l'existence, de la pensée et de l'action et qui fait naitre un engagement sociétal.

Y a-t-il quelque chose que vous souhaiteriez de votre association l'ASP?

Je souhaiterais que l'ASP s'engage davantage dans le domaine sociétal et politique, par ex. dans des consultations législatives ou par un travail d'explication ou de prises de position. Deux grands domaines importants seraient la formation scolaire et la psychologie pédagogique. Même si la psychothérapie est en soi exempte d'évaluation, je suis d'avis quelle doit assumer avec sa connaissance une responsabilité dans la société.

J'ai été par ex. ravie que l'association ait soutenu l'initiative et la pétition "Référendum contre la surveillance des assurés » avec la publication de la campagne sur son site Internet. Je souhaiterais voir plus souvent et davantage ce genre d'action, une prise de position active aussi dans les médias publics et peut-être aussi dêtre elle-même à l'origine d'une initiative.

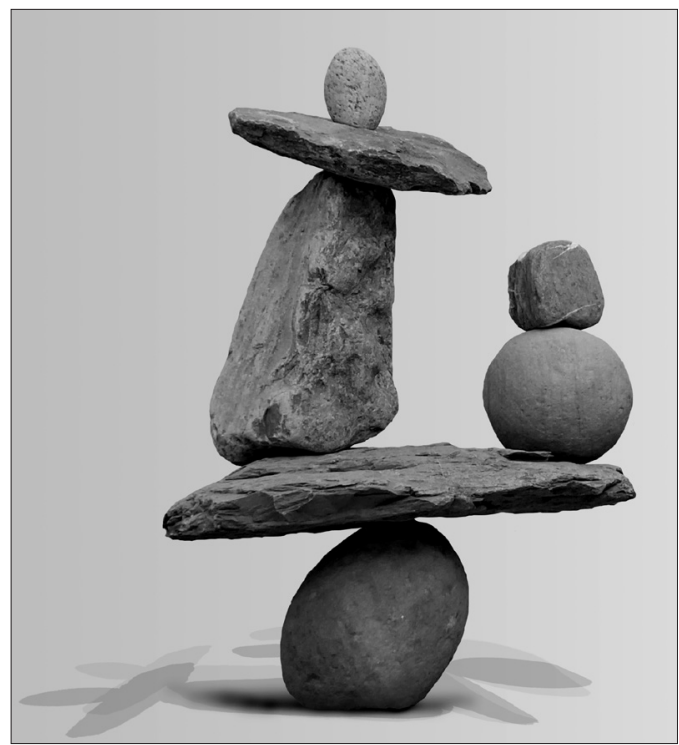

Je souhaite que l'association soutienne encore la voie de la reconnaissance de la diversité des méthodes et continue à la parcourir. Cette voie caractérise le mieux pour moi le travail de l'association pendant toutes ces années de son action.

Vous sentez-vous représentée et appréciée dans votre association professionnelle, l'ASP ?

Je me sens représentée et appréciée par mon association professionnelle, l'ASP. Je suis très reconnaissante pour le soutien que j'ai reçu de la part de l'association lorsque je suis arrivée en Suisse et lorsqu'il s'est agi de reconnaitre ma formation en Allemagne et en Suisse. Contrairement à beaucoup d'autres organismes avec lesquels j’ai été en contact, je l'ai ressenti avec chacune des personnes de l'ASP comme quelque chose de simple, accompagnant et ouvert. Cela m'avait à l'époque fortement impressionnée et aidée. Le fait aussi qu'il existe une association professionnelle qui reconnait la psychothérapie corporelle comme une méthode thérapeutique de la psychothérapie.

Je ne suis pas fervente de la préconisation du modèle de la prescription médicale, même si je comprends les motivations de lassociation. Je pense que les psychothérapeutes disposent de compétences tellement bonnes qu'ils doivent être reconnus de manière indépendante comme une profession et qu'ils doivent aussi agir. Et que cela doit être fait maintenant en ces temps de changements dans ce domaine. À mon sens, l'impulsion d'un nouveau changement deman- 
dera beaucoup de force et de temps une fois que le concept de la prescription médicale sera établi.

Je trouve que l'association fait un travail super avec la réalisation du concept transméthodique de la formation post-graduée en psychothérapie ASP Integral et je souhaiterais la remercier pour cela, même si je ne peux pas en profiter en raison de ma formation en biosynthèse. Je suis toutefois heureuse que l'on aide d'autres petites écoles de formation de cette façon à conserver leur reconnaissance et ainsi à pouvoir continuer à exister.

Quelle serait votre priorité si vous étiez dans le conseil d'administration de l'ASP?

Clairement les relations publiques dans le domaine politique et juridique et un travail explicatif dans la population. Vraisemblablement, je me concentrerais spécialement sur le secteur du trauma afin d'aider à la mise en réseau et à soutenir aussi des initiatives et activités déjà existantes, par ex. par un travail médiatique.

Y a-t-il une fonction dans l'ASP que vous occuperiez volontiers?

Je remercie tous ceux qui donnent leur temps et leurs forces dans le travail de l'association. En ce qui me concerne, je ne vois actuellement aucune capacité pour occuper un poste dans l'ASP, bien que la politique professionnelle m'intéresse beaucoup et que je la considère comme étant un travail très important.

Comment verriez-vous votre situation idéale dans un environnement politique donné pour les psychothérapeutes?

Les psychothérapeutes devraient être reconnus comme un groupe professionnel indépendant et pouvoir agir en toute indépendance. Je vois aussi des droits et obligations qui y sont associés. $\mathrm{Au}$ niveau des obligations, jintègre aussi le fait de s'engager politiquement et sur le plan sociétal et d'utiliser activement le savoir pour organiser notre vie et l'avenir des générations suivantes. Nous leur laissons des problèmes graves en dépit de notre bon sens. Je peux comprendre qu'au niveau politique et des caisses maladie, on a peur de l'expansion quantitative en ce qui concerne la prestation en psychothérapie. Pour moi, cela représente toutefois un conflit sociétal global dans lequel la rentabilisation et la croissance constante sont l'objectif ultime.
Quelle est votre vision dans votre quotidien professionnel?

Clairement que le métier de psychothérapeute soit reconnu comme groupe professionnel indépendant, tout comme le psychiatre est un groupe professionnel reconnu. Je suis moi-même médecin et estime énormément le travail des collègues actifs en psychiatrie, s'ils voulaient toutefois percevoir et comprendre la psychothérapie aussi largement que le font les psychologues, ou les spécialistes en médecine psychosomatique et en psychothérapie, ils devraient suivre en plus une formation de la même ampleur et pratiquer le savoir en permanence, comme nous.

Que cela soit aussi reconnu comme tel par le système sociétal et politique. Sur des formulaires suisses d'assurances d'indemnités journalières est écrit par ex. en gras qu'ils exigent impérativement la signature d'un psychiatre pour l'estimation.

Ce que l'on comprend c'est que plus de formalités et documentations, de mesures d'assurance-qualité, etc. signifient une énorme exploitation de ressources humaines hautement qualifiées et que cela nous détourne de notre travail réel.

Ma vision est que les psychothérapeutes se valorisent avec leur savoir et leurs compétences si fortement qu'ils soient pleinement conscients de leur signification dans le contexte sociétal. Je crois réellement quêtre psychothérapeute est une vocation.

Je souhaite fortement qu'avec mon rapport très personnel, je suscite la réflexion auprès d'autres personnes et peut-être que je pourrais inspirer l'une ou l'autre.

Je remercie du fond du cœur pour cette opportunité d'interview !

\section{Concernant la personne}

Esther Bulang, Dr med., est une psychothérapeute de l'ASP reconnue au niveau fédéral et une spécialiste en ophtalmologie (FMH) ainsi que spécialiste en médecine psychosomatique et psychothérapie. Elle est membre de l'ASP depuis 2013.

Son activité en cabinet: Assistante médicale et psychothérapeute auprès du Dr med. Heidrun Kurz, Wotanstrasse 10, 8032 Zurich, ainsi que dans son propre cabinet avec Madame Suna Baldinger, psychologue SBAP, Limmatstrasse 107, 8005 Zurich estherbulang@gmail.com

Cet interview a été mené par écrit par Veronica Defièbre. 